\title{
Pharmacokinetic parameters and radiomics model based on dynamic contrast enhanced MRI for the preoperative prediction of sentinel lymph node metastasis in breast cancer
}

Meijie Liu ${ }^{1,2+}$, Ning Mao ${ }^{2 \dagger}$, Heng Ma², Jianjun Dong ${ }^{2}$, Kun Zhang ${ }^{2}$, Kaili Che ${ }^{2}$, Shaofeng Duan ${ }^{3}$, Xuexi Zhang ${ }^{3}$, Yinghong $\mathrm{Shi}^{2^{*}}$ and Haizhu Xie ${ }^{2^{*}}$ (D)

\begin{abstract}
Background: To establish pharmacokinetic parameters and a radiomics model based on dynamic contrast enhanced magnetic resonance imaging (DCE-MRI) for predicting sentinel lymph node (SLN) metastasis in patients with breast cancer. Methods: A total of 164 breast cancer patients confirmed by pathology were prospectively enrolled from December 2017 to May 2018, and underwent DCE-MRI before surgery. Pharmacokinetic parameters and radiomics features were derived from DCE-MRI data. Least absolute shrinkage and selection operator (LASSO) regression method was used to select features, which were then utilized to construct three classification models, namely, the pharmacokinetic parameters model, the radiomics model, and the combined model. These models were built through the logistic regression method by using 10 -fold cross validation strategy and were evaluated on the basis of the receiver operating characteristics (ROC) curve. An independent validation dataset was used to confirm the discriminatory power of the models.

Results: Seven radiomics features were selected by LASSO logistic regression. The radiomics model, the pharmacokinetic parameters model, and the combined model yielded area under the curve (AUC) values of 0.81 (95\% confidence interval [Cl]: 0.72 to 0.89$), 0.77$ (95\% Cl: 0.68 to 0.86 ), and 0.80 ( $95 \%$ Cl: 0.72 to 0.89 ), respectively, for the training cohort and 0.74 ( $95 \%$ Cl: 0.59 to 0.89 ), 0.74 ( $95 \%$ Cl: 0.59 to 0.90 ), and 0.76 ( $95 \%$ Cl: 0.61 to 0.91 ), respectively, for the validation cohort. The combined model showed the best performance for the preoperative evaluation of SLN metastasis in breast cancer.
\end{abstract}

Conclusions: The model incorporating radiomics features and pharmacokinetic parameters can be conveniently used for the individualized preoperative prediction of SLN metastasis in patients with breast cancer.

Keywords: Breast cancer, Sentinel lymph node, Magnetic resonance imaging, Radiomics, Pharmacokinetic parameters

\footnotetext{
*Correspondence: shyhyt@163.com; xhz000417@sina.com

${ }^{\dagger}$ Meijie Liu and Ning Mao contributed equally to this work.

${ }^{2}$ Department of Radiology, Yantai Yuhuangding Hospital, No. 20

Yuhuangding road, Yantai, Shandong, P. R. China 264000

Full list of author information is available at the end of the article
}

C C The Author(s). 2020 Open Access This article is licensed under a Creative Commons Attribution 4.0 International License, which permits use, sharing, adaptation, distribution and reproduction in any medium or format, as long as you give appropriate credit to the original author(s) and the source, provide a link to the Creative Commons licence, and indicate if changes were made. The images or other third party material in this article are included in the article's Creative Commons licence, unless indicated otherwise in a credit line to the material. If material is not included in the article's Creative Commons licence and your intended use is not permitted by statutory regulation or exceeds the permitted use, you will need to obtain permission directly from the copyright holder. To view a copy of this licence, visit http://creativecommons.org/licenses/by/4.0/ The Creative Commons Public Domain Dedication waiver (http://creativecommons.org/publicdomain/zero/1.0/) applies to the data made available in this article, unless otherwise stated in a credit line to the data. 


\section{Background}

Breast cancer is a common malignancy in women and a major cause of cancer deaths [1]. Axillary lymph node status is one of the strongest prognostic factors in patients with breast cancer and is crucial for the treatment of this disease [2]. Since the 1990s, the sentinel lymph node biopsy (SLNB) for breast cancer has replaced axillary lymph node dissection (ALND) as the standard of care for primary treatment of early breast cancer [3]. However, this method is invasive and carries the risk of dye allergies and false negative results [4]. Therefore, a noninvasive and accurate method for the detection of sentinel lymph node (SLN) metastasis would be crucial in avoiding unnecessary postoperative complications and selecting the optimal therapy in clinical practice.

Breast cancer generally features distinct histological, molecular, and clinical phenotypes and may manifest as radiologic heterogeneity. Radiomics can transform image data into high-resolution image feature data that can be mined and provide deep quantitative information that cannot be determined by the naked eye of clinicians [5]. Radiomics is predictive of malignancy, response to neoadjuvant chemotherapy, prognostic factors, molecular subtypes, and recurrence risk in breast cancer [6,7] and thus shows promising use in assessing and predicting SLN metastasis in tumors $[8,9]$.

Magnetic resonance imaging (MRI) has been widely used in the diagnosis and staging of breast cancer because of its advantages of non-radiation, high soft tissue contrast and functional imaging. Although studies have showed that MRI has superior performance than some other techniques such as ultrasound or mammography, its efficacy in identifying axillary lymph node status is unsatisfactory $[10,11]$. Dynamic contrast enhanced magnetic resonance imaging (DCE-MRI) can provide pharmacokinetic parameters, including semiquantitative and quantitative parameters, and is a sensitive technique that reflects the extent of tumor angiogenesis [12]. Previous studies have demonstrated that pharmacokinetic parameters can potentially be used as prognostic or predictive biomarkers [13]. Bahri et al. [14] found that the Ktrans and Kep values of metastatic lymph nodes are higher than those of nonmetastatic lymph nodes in breast cancer. However, using combined pharmacokinetic parameters and DCE-MRI radiomics features to predict SLN metastasis has not yet been demonstrated.

Therefore, the aim of our study was to construct and validate a noninvasive model from preoperative DCE-MRI to predict SLN metastasis in patients with breast cancer.

\section{Methods}

\section{Patients}

This prospective study was approved by the ethics committee, and written informed consent was obtained from all patients. We identified 257 consecutive patients with newly histologically proven invasive breast cancer from December 1, 2017 to May 1, 2018. The inclusion criteria were 1) had undergone pathological evaluation of SLN; 2) cancer focus with longest diameter $>5 \mathrm{~mm}$; and 3) single mass enhancement. Exclusion criteria were 1) nonmass-like enhancement on DCE-MR images; 2) incomplete clinical or pathologic characteristics; and 3) undergone radiation therapy or chemotherapy treatment. In this study, all cases underwent axilla ultrasound. Patients with negative axilla by ultrasound underwent SLNB at the time of surgery. If the SLN was positive, ALND was performed. If patient was found to have suspicious positive axillary lymph node by ultrasound, an ultrasound-guided fine needle biopsy was performed. If there was a histologically positive lymph node on needle biopsy, the patient received ALND. If the biopsy result was negative, the patient received a SLNB.

Finally, 164 patients (78 positive for SLN and 86 negative for SLN) were included in this study. The training cohort included 124 cases from December 2017 to March 2018, and the validation cohort included 40 cases from April 2018 to May 2018.

\section{MRI acquisition}

All images were obtained with a $3.0 \mathrm{~T}$ MRI system (GE Discovery $750 \mathrm{~W}$ ) using an 8-channel breast-dedicated coil in prone position. The MRI sequences included axial T1weighted imaging (T1WI) (repetition time [TR]/echo time $[\mathrm{TE}]=520 \mathrm{~ms} / 9 \mathrm{~ms}$, slice thickness $/$ gap $=5 \mathrm{~mm} / 1 \mathrm{~mm})$ and axial T2-weighted imaging (T2WI) (TR/TE $=5200 \mathrm{~ms} / 90$ $\mathrm{ms}$, slice thickness/gap $=5 \mathrm{~mm} / 1 \mathrm{~mm})$. DCE-MRI was performed with volume acceleration sequence in the axial plane $(\mathrm{TR} / \mathrm{TE}=6.2 \mathrm{~ms} / 2.3 \mathrm{~ms}$; slice thickness $/$ gap $=2$ $\mathrm{mm} / 0 \mathrm{~mm} ; \quad$ FOV $=360 \times 360 \mathrm{~mm}^{2} ;$ matrix $=288 \times 320$; and flip angles: $5^{\circ}, 10^{\circ}$, and $15^{\circ}$ ). The contrast agent (Omniscan, $0.2 \mathrm{mmol} / \mathrm{kg}$ body) was injected after the acquisition of one set of precontrast images by using a high-pressure syringe at a rate of $2.8 \mathrm{ml} / \mathrm{s}$, followed by the injection of equivalent volume of saline at the same rate to wash out the residual contrast agent in the tube. The scanning time of each phase was $16 \mathrm{~s}$ for a total of 30 phases. In this study, we used the peak enhanced phase of the multiphase contrast-enhanced MRI selected in accordance with the time intensity curve because the image lesions had the largest amount of contrast with the background [15].

\section{Pharmacokinetic parameter extraction}

DCE-MRI data were transferred to an off-line workstation and analyzed by using specialized quantitative analysis software (Omni Kinetics; GE Healthcare, China, Shanghai). Two experienced radiologists (with 10 years of experience in breast imaging diagnosis) who were blinded to the histopathological results independently 
performed the data analysis. Before parameter calculation, a nonlinear registration framework utilizing the Free Form Deformation algorithm was used to correct misalignment caused by body motion between consecutive DCE scans, and signal intensity was converted into omniscan concentration using the variable flip angle method. An arterial input function was extracted by manually drawing on the thoracic aorta. The two-compartment extended Tofts model was selected to calculate pharmacokinetic parameters, including the quantitative parameters volume transfer constant (Ktrans), reverse reflux rate constant (Kep), volume fraction of extravascular extracellular space $(\mathrm{Ve})$, and volume fraction of plasma $(\mathrm{Vp})$, as well as semiquantitative parameters, including time to peak (TTP), maximum concentration (MaxCon), maximal slope (MaxSlope), and area under curve (AUC). The entire tumor maximum layer was selected as the region of interest (ROI), and necrotic or cystic areas were excluded from the evaluation.

\section{Radiomics feature extraction}

Prior to image feature extraction, the original MRI image must be preprocessed; preprocessing steps include MRI signal intensity standardization and Gray-level quantization $[16,17]$. On the basis of time signal intensity curves, the strongest enhanced phase was selected and the ROI was manually drawn by a radiologist with more than 10 years of experience and who was blinded to the pathological results (Fig. 1). As shown in Table 1, a total of 396 radiomics features classified as first-order, shape, and texture features were extracted from the ROI. This process was performed with AK software (Artificial Intelligence Kit; GE Healthcare, China, Shanghai).

\section{Feature selection}

Intra- and inter-observer agreement was analyzed on the basis of intra- and inter-class correlation coefficient (ICCs) for all radiomics features extraction. A total of 30 random patients were selected, and radiologist $A$ and radiologist $B$ extracted the features of these 30 patients. Radiologist A then repeated the same procedure 1 week later. The ICCs greater than 0.75 indicated good consistency.

The LASSO logistic regression algorithm, which is suitable for the regression of high-dimensional data, was used to select the most useful predictive features from the primary data set.

\section{Predictive model building}

The enrolled cases were randomly divided into two independent subsets at a ratio of $3: 1$, wherein 124 patients were used as the training cohort, and 40 patients were used as the independent validation cohort.

The logistics regression models were used to establish the radiomics model, the pharmacokinetic parameters model, and the combined model to predict SLN metastasis in breast cancer. The predictive efficiency of the predictive model was assessment by using receiver operating characteristics (ROC) curve in the training and validation cohorts.

\section{Statistical analysis}

Patients age and tumor diameter were compared between the SLN-positive and SLN-negative groups by $t$ test. Histological grade and molecular subtype were tested for trends using the chi-square test. All numerical data were presented as mean standard deviation. LASSO logistic regression was performed on the basis of 10-fold cross validation. Multivariate logistic regression was used to develop three models: the radiomics model, pharmacokinetic

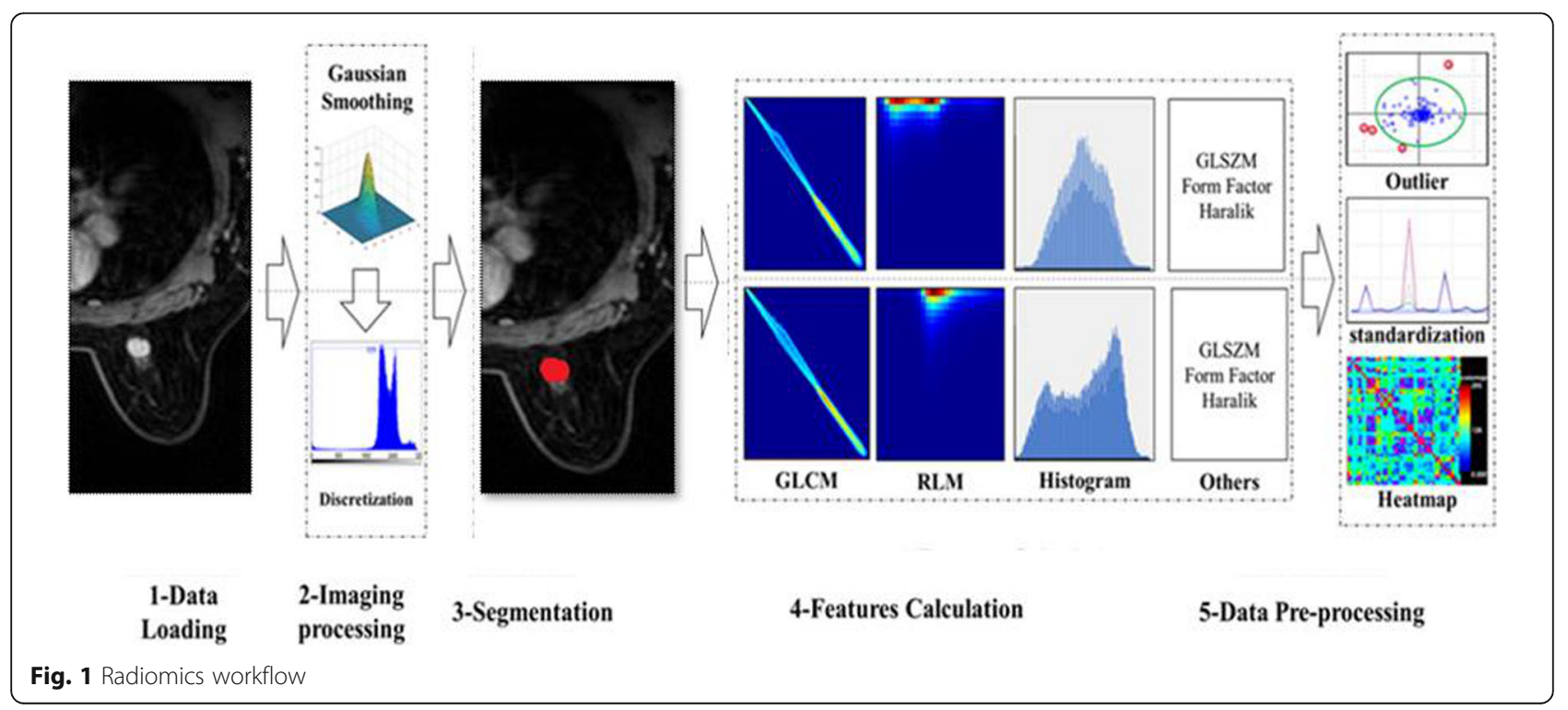


Table 1 Radiomic features derived from the images

\begin{tabular}{lll}
\hline $\begin{array}{l}\text { Calculation } \\
\text { Methods }\end{array}$ & Radiomics Features & $\begin{array}{l}\text { Feature } \\
\text { Numbers }\end{array}$ \\
\hline Histogram & Frequency size, Quantile, Variance, Kurtosis, Skewness, etc. & 42 \\
GLSZM & Size Zone Variability, Large Area Emphasis, High Intensity Emphasis etc. & 11 \\
Haralick matrix & HaraEntroy, Contrast, Inverse Difference Moment, Sum Average, Sum Variance & 10 \\
Form factor matrix & Maximum 3D Diameter, Spherical Disproportion, Sphericity, Surface Area, etc. & 9 \\
GLCM & ClusterProminence_AllDirection_offset1, Correlation_AllDirection_offset1, GLCMEnergy_angle45_offset4, etc. & 144 \\
RLM & Grey Level Non-Uniformity All Direction, High Grey Level Run Emphasis Angle Offset, Run Length Non- & 180 \\
& uniformity Angle Offset, etc. &
\end{tabular}

parameters model, and the combined model. ROC curve were used to evaluate the diagnostic performance of the three models. Sensitivity, specificity, and accuracy were calculated for each model. A two-sided $p$ value of less than 0.05 was considered to indicate significant difference. All statistical tests were carried out in R3.5.1.

\section{Results}

\section{Clinical characteristics}

As shown in Table 2, age, tumor diameter, histological grade, and molecular subtype did not significantly differ between the SLN-positive and SLN-negative groups $(P>0.05)$.

\section{Feature selection and predictive performance of the model}

The intra-observer ICC ranged from 0.869 to 0.894 , and the inter-observer ICC ranged from 0.851 to 0.926 , indicating favorable feature extraction reproducibility.

The training cohort had 396 selected radiomics features for LASSO logistic regression analysis. A total of seven radiomics features including Sum Entropy, Compactness1, Inertia, Cluster Prominence, Correlation, GLCM Entropy, Kurtosis were selected by the LASSO logistic regression model (Fig. 2). SLN metastasis prediction models were developed using a multivariate logistic regression model based on pharmacokinetic parameters (Ktrans,
Kep, Ve, Vp, TTP, MaxSlope, AUC, and MaxCon) and radiomics features.

In the training cohort, the radiomics model, pharmacokinetic parameters model, and the combined model yielded AUC values of 0.81 (95\% CI: 0.72 to 0.89 ), 0.77 (95\% CI: 0.68 to 0.86 ), and 0.80 (95\% CI: 0.72 to 0.89 ), respectively. In the validation cohort, the pharmacokinetic parameters model, the radiomics model, and the combined model yielded AUC values of 0.74 (95\% CI: 0.59 to 0.89 ), 0.74 (95\% CI: 0.59 to 0.90$)$, and 0.76 (95\% CI: 0.61 to 0.91 ), respectively (Fig. 3 ).

The results for the validation cohort showed that the accuracy, sensitivity, and specificity of the pharmacokinetic parameters model were 69,71 , and $77 \%$, respectively. The accuracy, sensitivity, and specificity of the radiomics model were 67,64 and $79 \%$, respectively. The accuracy, sensitivity, and specificity of the combined model were 76 , 72 and $81 \%$, respectively. The combined model results showed that the effect was higher than another (Table 3).

\section{Discussion}

In this study, the multivariate logistic regression model was used to establish prediction models for predicting SLN metastasis. The results showed that the combination of radiomics and hemodynamic characteristics can obtain an improved preoperative prediction model with

Table 2 Clinical and Histopathological Characteristics

\begin{tabular}{|c|c|c|c|}
\hline & Patients with positive SLN $(n=78)$ & Patients with negative $\operatorname{SLN}(n=86)$ & $p$ value \\
\hline Age (mean $\pm S D)$ & $55.71 \pm 8.6$ & $54.40 \pm 11.1$ & 0.483 \\
\hline Tumor size (mean $\pm \mathrm{SD}$ ) & $2.24 \pm 1.0$ & $2.21 \pm 1.2$ & 0.698 \\
\hline Histological grade & & & 0.171 \\
\hline I & $4(5.15 \%)$ & $25(11.6 \%)$ & \\
\hline$\|$ & $33(42.3 \%)$ & $41(47.7 \%)$ & \\
\hline III & $41(52.6 \%)$ & $20(40.7 \%)$ & \\
\hline Molecular subtype & & & 0.220 \\
\hline Luminal A & $30(38.5 \%)$ & $31(36 \%)$ & \\
\hline Luminal B & $37(47.4 \%)$ & $33(38.4 \%)$ & \\
\hline HER2 over-expression & $6(7.7 \%)$ & $8(9.3 \%)$ & \\
\hline Basal-like & $5(6.4 \%)$ & $14(16.3 \%)$ & \\
\hline
\end{tabular}


a

$\begin{array}{llllllllllllllllllllll}11 & 11 & 12 & 13 & 12 & 11 & 11 & 11 & 11 & 11 & 11 & 11 & 10 & 8 & 8 & 7 & 7 & 7 & 7 & 6 & 4 & 3\end{array}$
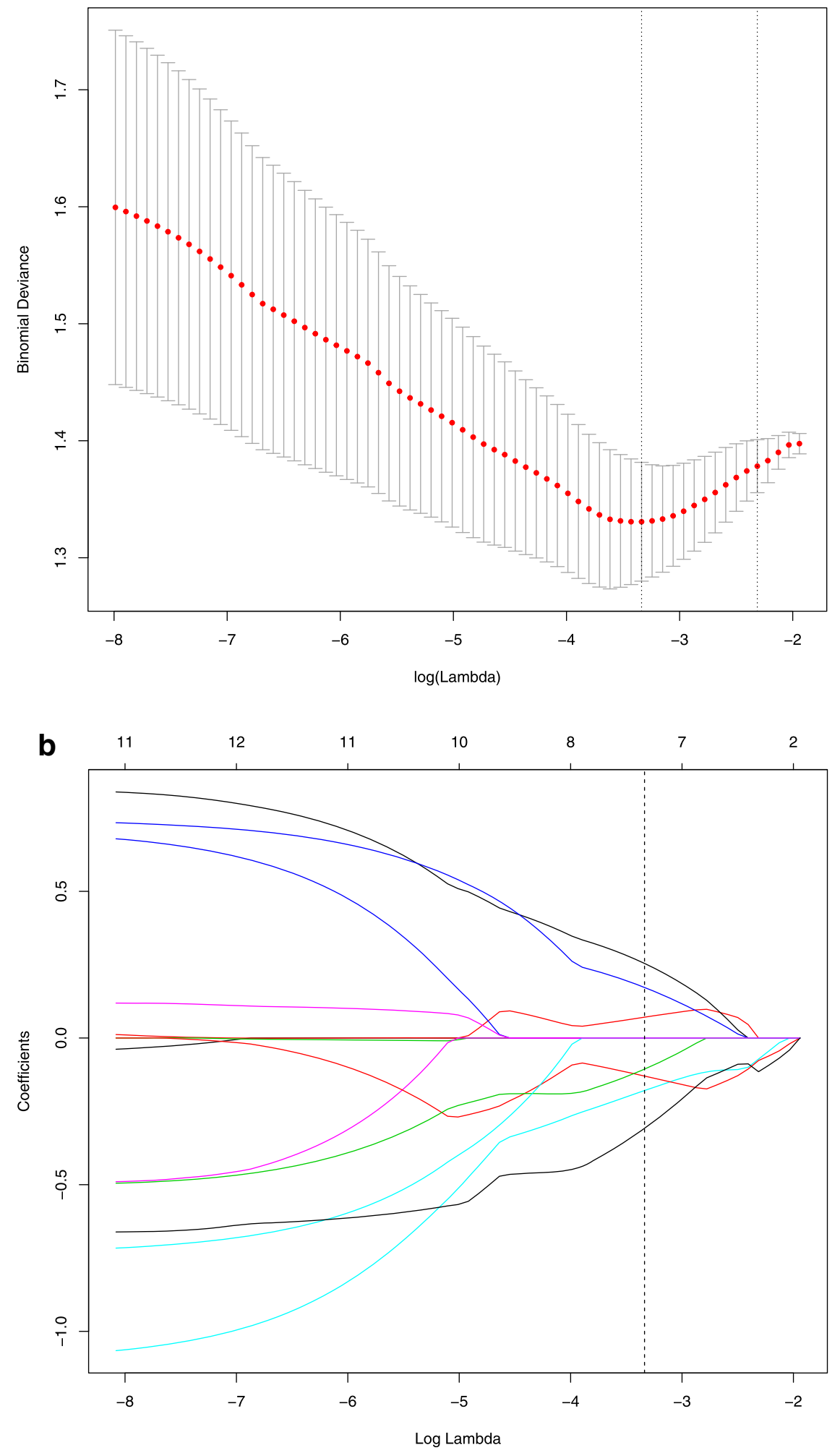

Fig. $\mathbf{2}$ LASSO algorithm for feature selection. a Selection of adjustment parameters (lambda) in the LASSO model used 10-fold cross-validation via minimum criteria; $\mathbf{b}$ LASSO coefficient profiles of the features against the $\log (\lambda)$ 
promising performance in the prediction of SLN metastases with an AUC of 0.76 in the validation set. These results might help clinical decision-making with respect to axillary surgery, potentially avoiding invasive procedures in patients at a low risk of SLN metastases.

Assessing axillary lymph node metastasis as early as possible is essential for breast cancer surgical planning, adjuvant therapy planning, and prognostication. Determining axillary lymph node status remains a mandatory requirement of diagnostics. The SLNB and ALND are common methods for the estimation of axillary lymph node status. However, they are invasive [18]. Therefore, a noninvasive approach with high accuracy is necessary to preoperatively evaluate SLN metastasis. Developing a tool that accurately and noninvasively predicts axillary lymph node metastasis preoperatively provides great advantage. Yang used the radiomics of mammography to preoperatively predict SLN metastasis [19]. However, X-ray is limited by difficult detection of the complete picture of the lesion, the easy overlap between the lesion site and glandular tissue, and the easily missed diagnosis. MRI can completely show the type, range, and internal structure of lesions and clearly show the multicenter lesions. At the same time, DCE-MRI has a good diagnostic effect for tumor recurrence foci and multicentric cancer [20].

DCE-MRI can provide multiple pharmacokinetic parameters, including semiquantitative parameters and quantitative parameters, which can reveal the perfusion and vascular distribution of tissue at the molecular level [21]. The DCE-MRI-derived parameters Ktrans and Kep enable the estimation of tumor angiogenesis and proliferation in breast cancer [22]. Previous studies show that
Table 3 Diagnostic performance of validation cohort

\begin{tabular}{llll}
\hline & accuracy & sensitivity & specificity \\
\hline pharmacokinetic parameters model & 0.69 & 0.71 & 0.77 \\
Radiomics model & 0.67 & 0.64 & 0.79 \\
Combined model & 0.76 & 0.72 & 0.81 \\
\hline
\end{tabular}

quantitative parameters can improve diagnostic accuracy and provide insight into the underlying biological characteristics of breast lesions [23]. Compared with semiquantitative parameters, quantitative parameters are less affected by the wide variability in MRI scanners, scanning sequence, temporal resolution, contrast media injection, and image postprocessing calculation [24]. In this study, we combined quantitative parameters (Ktrans, Kep, Ve, and $\mathrm{Vp}$ ) with semiquantitative parameters (TTP, MaxSlope, AUC, and MaxCon), and demonstrated satisfactory performance in the diagnosis of SLN metastasis with an AUC of 0.77 in the training dataset and AUC of 0.74 in the validation dataset; these results indicate that the good effect of our model for the differentiation of SLN metastasis is better than that of previously reported methods [25].

Radiomics, an emerging technique that can convert digital medical images into mineable data for analysis for texture feature extraction, helps characterization within heterogeneous tumor lesions [26]. This approach can help clinicians improve detection, diagnosis, stage, and prediction power. Only a few studies have shown that DCE-MRI can predict the occurrence of SLN metastasis with high accuracy by radiomics $[27,28]$. In 2018 , Liu first attempted to predict SLN metastasis in breast cancer noninvasively by using DCE-MRI radiomics and
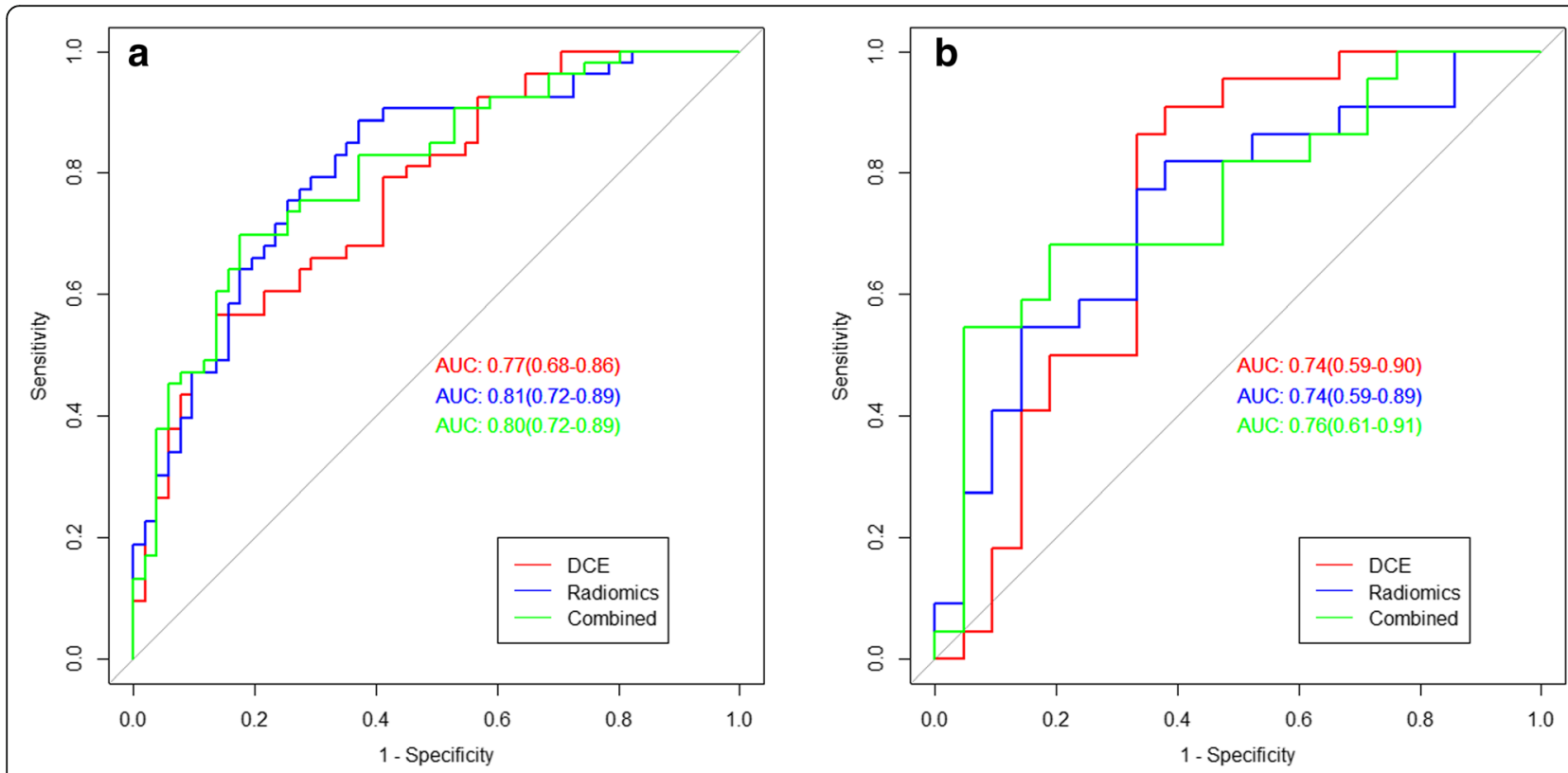

Fig. 3 ROC curves of prediction models in the training (a) and validation (b) cohorts 
demonstrated promising prediction performance in an independent validation set [29]. However, this study was retrospectively designed, whereas our study was a prospective study with radiomics from breast cancer. Our study sequence was standardized, and our evidence for clinical application was stringent. Previous studies only used DCE-MRI for feature extraction, and the accuracy of the comprehensive analysis of pharmacokinetic parameters was unknown. In our study, the model that combined radiomics features and pharmacokinetic parameters with an AUC of 0.76 in the validation cohort had higher prediction ability than any single model. The combined model indicates microcirculation function and information regarding tissue morphological characteristics, which can provide a comprehensive description of the tumor.

Recently, radiomics analysis based on other MRI sequences has been reported. In 2017, Dong applied T2-WI and DWI texture features to predict SLN metastasis [30]. Most of lesions on DWI and T2W images show reduced resolution and exacerbated distortion, and segmenting the lesions completely is difficult. In this study, DCE-MRI was used to derive DCE parameters and radiomics analysis, which clearly show the lesion boundaries. DCE-MRI has numerous scanning phases. Han et al. [28] extracted radiomics features from the axial first phase of the T1weighted images of the DCE images of primary tumors to predict SLN metastasis. In this study, we used the peak enhanced phase of multiphase contrast enhanced MRI selected in accordance with the time intensity curve, which show the lesion boundaries clearly and have the greatest association with potential tissue information [15].

This study has several limitations. First, it is a preliminary exploratory study with a small sample size, and a large sample is necessary. Second, the manual method was applied to image segmentation although the satisfactory inter- and intra-observer reproducibility of radiomics features extraction was achieved. Moreover, over $90 \%$ of features had good reproducibility [31]. However, the automated method for image segmentation may have high stability [32, 33]. Third, our study lack external validation for the model. Multicenter validation is needed to acquire high-level evidence for clinical application. We will continue our research in future studies.

\section{Conclusions}

In conclusion, our prospective research shows that pharmacokinetic parameters and the radiomics combined model represent a noninvasive predictive tool that shows good application prospects for the detection of SLN metastasis in patients with breast cancer. A multicenter validation study with a large sample size should be conducted to improve efficiency in subsequent works.

\section{Abbreviations}

DCE-MRI: Dynamic contrast enhanced magnetic resonance imaging; SLN: Sentinel lymph node; LASSO: Least absolute shrinkage and selection operator; Ktrans: Quantitative parameters volume transfer constant; Kep: Reverse reflux rate constant; Ve: Volume fraction of extravascular extracellular space; Vp: Volume fraction of plasma; TTP: Time to peak; MaxCon: Maximum concentration; MaxSlope: Maximal slope; ROC: Receiver operating characteristics; AUC: Area under curve; ROI: Region of interest; ICCs: Intra- and interclass correlation coefficient; Cl: Confidence interval

\section{Acknowledgements}

Not applicable.

\section{Authors' contributions}

HZX and YHS conceived the idea of the study. HM, JJD, KZ and KLC provided the data. SFD, XXZ, MJL and NM performed the statistical analysis. MJL and NM wrote the paper. All authors discussed the results and revised the final manuscript. The authors read and approved the final manuscript.

\section{Funding}

This study was supported by the Natural Science Foundation of China (81671654), Natural Science Foundation of Shandong Province of China (ZR2017PH043), Program for Medical Science and Technology of Shandong Province of China (2016WS0713), and Special Foundation for Sun Simiao Traditional Chinese Medicine of China Medicine Education Association (2016SKT-M034).

\section{Availability of data and materials}

The processed data required to reproduce these findings cannot be shared at this time as the data also forms part of an ongoing study.

Ethics approval and consent to participate

This study was approved by the medical ethics committee of Yantai Yuhuangding Hospital and all patients signed informed consents.

\section{Consent for publication}

Not applicable.

\section{Competing interests}

All authors are in agreement with the content of the manuscript. The manuscript is approved by all authors for publication.

\section{Author details}

${ }^{1}$ School of Clinical Medicine, Binzhou Medical University, Yantai, Shandong, P. R. China 264000. ²Department of Radiology, Yantai Yuhuangding Hospital, No. 20 Yuhuangding road, Yantai, Shandong, P. R. China 264000. ${ }^{3} \mathrm{GE}$ Healthcare, China, Shanghai, P. R. China 200000.

Received: 23 December 2019 Accepted: 2 September 2020 Published online: 15 September 2020

\section{References}

1. Miller KD, Siegel RL, Lin CC, Mariotto AB, Kramer JL, Rowland JH, et al. Cancer treatment and survivorship statistics, 2016. CA Cancer J Clin. 2016; 66(4):271-89.

2. Weigel MT, Dowsett M. Current and emerging biomarkers in breast cancer: prognosis and prediction. Endocr Relat Cancer. 2010;17(4):R245-62.

3. Lyman GH, Giuliano AE, Somerfield MR, Benson AB III, Bodurka DC, Burstein $\mathrm{HJ}$, et al. American Society of Clinical Oncology guideline recommendations for sentinel lymph node biopsy in early-stage breast cancer. J Clin Oncol. 2005;23(30):7703-20.

4. Chatterjee A, Serniak N, Czerniecki BJ. Sentinel lymph node biopsy in breast cancer: a work in progress. Cancer J (Sudbury, Mass). 2015;21(1):7.

5. Marusyk A, Almendro V, Polyak K. Intra-tumour heterogeneity: a looking glass for cancer? Nat Rev Cancer. 2012;12(5):323-34.

6. Mao N, Wang Q, Liu M, Dong J, Xiao C, Sun N, et al. Computerized image analysis to differentiate benign and malignant breast tumors on magnetic resonance diffusion weighted image: a preliminary study. J Comput Assist Tomogr. 2019;43(1):93-7.

7. Chamming's F, Ueno $Y$, Ferre R, Kao E, Jannot AS, Chong J, et al. Features from computerized texture analysis of breast cancers at pretreatment MR 
imaging are associated with response to Neoadjuvant chemotherapy. Radiology. 2018;286(2):412-20.

8. Zhong Y, Yuan M, Zhang T, Zhang YD, Li H, Yu TF. Radiomics approach to prediction of occult Mediastinal lymph node metastasis of lung adenocarcinoma. AJR Am J Roentgenol. 2018;211(1):109-13.

9. Mao N, Yin P, Li Q, Wang Q, Liu M, Ma H, et al. Radiomics nomogram of contrast-enhanced spectral mammography for prediction of axillary lymph node metastasis in breast cancer: a multicenter study. Eur Radiol. 2020. https://doi.org/10.1007/s00330-020-07016-z.

10. Saidha NK, Aggarwal R, Sen A. Identification of sentinel lymph nodes using contrast-enhanced ultrasound in breast Cancer. Indian J Surg Oncol. 2018; 9(3):355-61.

11. Diepstraten SC, Sever AR, Buckens CF, Veldhuis WB, van Dalen T, van den Bosch MA, et al. Value of preoperative ultrasound-guided axillary lymph node biopsy for preventing completion axillary lymph node dissection in breast cancer: a systematic review and meta-analysis. Ann Surg Oncol. 2014 21(1):51-9.

12. Thomas AA, Arevalo-Perez J, Kaley T, Lyo J, Peck KK, Shi W, et al. Dynamic contrast enhanced T1 MRI perfusion differentiates pseudoprogression from recurrent glioblastoma. J Neuro-Oncol. 2015;125(1):183-90.

13. Pieper CC, Sprinkart AM, Meyer C, König R, Schild HH, Kukuk GM, et al. Evaluation of a simplified intravoxel incoherent motion (IVIM) analysis of diffusion-weighted imaging for prediction of tumor size changes and imaging response in breast cancer liver metastases undergoing radioembolization: a retrospective single center analysis. Medicine. 2016; 95(14):e3275.

14. Bahri S, Chen J-H, Yu H, Kuzucan A, Nalcioglu O, Su M-Y. Can dynamic contrast-enhanced MRI (DCE-MRI) predict tumor recurrence and lymph node status in patients with breast cancer? Ann Oncol. 2008;19(4):822-4.

15. Liu Z, Li Z, Qu J, Zhang R, Zhou X, Li L, et al. Radiomics of multiparametric MRI for pretreatment prediction of pathologic complete response to Neoadjuvant chemotherapy in breast Cancer: a multicenter study. Clin Cancer Res. 2019;25(12):3538-47.

16. Mayerhoefer ME, Szomolanyi P, Jirak D, Materka A, Trattnig S. Effects of MRI acquisition parameter variations and protocol heterogeneity on the results of texture analysis and pattern discrimination: an application-oriented study. Med Phys. 2009;36(4):1236-43.

17. Gibbs P, Turnbull LW. Textural analysis of contrast-enhanced MR images of the breast. Magn Reson Med. 2003;50(1):92-8.

18. DiSipio T, Rye S, Newman B, Hayes S. Incidence of unilateral arm lymphoedema after breast cancer: a systematic review and meta-analysis. Lancet Oncol. 2013;14(6):500-15.

19. Yang J, Wang T, Yang L, Wang Y, Li H, Zhou X, et al. Preoperative prediction of axillary lymph node metastasis in breast Cancer using mammographybased Radiomics method. Sci Rep. 2019;9(1):4429.

20. Sardanelli F, Boetes C, Borisch B, Decker T, Federico M, Gilbert FJ, et al. Magnetic resonance imaging of the breast: recommendations from the EUSOMA working group. Eur J Cancer. 2010;46(8):1296-316.

21. Lollert A, Junginger T, Schimanski CC, Biesterfeld S, Gockel I, Duber C, et al, Rectal cancer: dynamic contrast-enhanced MRI correlates with lymph node status and epidermal growth factor receptor expression. J Magn Reson Imaging. 2014;39(6):1436-42.

22. Li L, Wang K, Sun X, Wang K, Sun Y, Zhang G, et al. Parameters of dynamic contrast-enhanced MRI as imaging markers for angiogenesis and proliferation in human breast cancer. Med Sci Monit. 2015;21:376-82.

23. Oshida K, Nagashima T, Ueda T, Yagata H, Tanabe N, Nakano S, et al. Pharmacokinetic analysis of ductal carcinoma in situ of the breast using dynamic MR mammography. Eur Radiol. 2005;15(7):1353-60.

24. Jansen SA, Fan X, Karczmar GS, Abe H, Schmidt RA, Newstead GM. Differentiation between benign and malignant breast lesions detected by bilateral dynamic contrast-enhanced MRI: a sensitivity and specificity study. Magn Reson Med. 2008;59(4):747-54

25. Liu Z, Feng B, Li C, Chen Y, Chen Q, Li X, et al. Preoperative prediction of lymphovascular invasion in invasive breast cancer with dynamic contrastenhanced-MRI-based radiomics. J Magn Reson Imaging. 2019;50(3):847-57.

26. Lambin P, Rios-Velazquez E, Leijenaar R, Carvalho S, van Stiphout RG, Granton $P$, et al. Radiomics: extracting more information from medical images using advanced feature analysis. Eur J Cancer. 2012:48(4):441-6.

27. Liu J, Sun D, Chen L, Fang Z, Song W, Guo D, et al. Radiomics analysis of dynamic contrast-enhanced magnetic resonance imaging for the prediction of sentinel lymph node metastasis in breast Cancer. Front Oncol. 2019;9:980.
28. Han L, Zhu Y, Liu Z, Yu T, He C, Jiang W, et al. Radiomic nomogram for prediction of axillary lymph node metastasis in breast cancer. Eur Radiol. 2019;29(7):3820-9

29. Liu C, Ding J, Spuhler K, Gao Y, Serrano Sosa M, Moriarty M, et al. Preoperative prediction of sentinel lymph node metastasis in breast cancer by radiomic signatures from dynamic contrast-enhanced MRI. J Magn Reson Imaging. 2019;49(1):131-40.

30. Dong Y, Feng Q, Yang W, Lu Z, Deng C, Zhang L, et al. Preoperative prediction of sentinel lymph node metastasis in breast cancer based on radiomics of T2-weighted fat-suppression and diffusion-weighted MRI. Eur Radiol. 2018;28(2):582-91.

31. Lee M, Woo B, Kuo MD, Jamshidi N, Kim JH. Quality of Radiomic features in Glioblastoma Multiforme: impact of semi-automated tumor segmentation software. Korean J Radiol. 2017;18(3):498-509.

32. Jung SC, Choi SH, Yeom JA, Kim JH, Ryoo I, Kim SC, et al. Cerebral blood volume analysis in glioblastomas using dynamic susceptibility contrastenhanced perfusion MRI: a comparison of manual and semiautomatic segmentation methods. PLoS One. 2013;8(8):e69323.

33. de Hoop B, Gietema H, van Ginneken B, Zanen P, Groenewegen G, Prokop M. A comparison of six software packages for evaluation of solid lung nodules using semi-automated volumetry: what is the minimum increase in size to detect growth in repeated CT examinations. Eur Radiol. 2009;19(4): $800-8$.

\section{Publisher's Note}

Springer Nature remains neutral with regard to jurisdictional claims in published maps and institutional affiliations.
Ready to submit your research? Choose BMC and benefit from:

- fast, convenient online submission

- thorough peer review by experienced researchers in your field

- rapid publication on acceptance

- support for research data, including large and complex data types

- gold Open Access which fosters wider collaboration and increased citations

- maximum visibility for your research: over $100 \mathrm{M}$ website views per year

At BMC, research is always in progress.

Learn more biomedcentral.com/submissions 